\title{
Bounds2: A Tool for Compositional Multi-parametrised Verification
}

\author{
Antti Siirtola \\ Helsinki Institute for Information Technology HIIT \\ Department of Computer Science and Engineering, Aalto University
}

\begin{abstract}
Bounds2 is a two-part tool for parametrised verification. The instance generator inputs a parametrised system implementation and specification, computes cut-offs for the values of the parameters and outputs the specification and implementation instances up to the cutoffs. After that, the outputted instances are verified by using an instance checker. Bounds2 is unique since it lends support to compositional reasoning through three refinement-based notions of correctness and allows for parametrising not only the number of processes but also the size of data types as well as the structure of a system. Bounds2 provides a sound and complete approach to parametrised verification under explicit assumptions checked automatically by the tool. The decidable fragment covers, e.g., mutual exclusion properties of systems with shared resources.
\end{abstract}

\section{Introduction}

Modern software systems are not only multithreaded but also object-oriented and component-based. Such systems have several natural parameters, such as the number of processes and the number of data objects. Moreover, some components, like external libraries and subsystems concurrently under construction, may only be available in the interface specification form. That is why there is an evident need for verification techniques that can handle multi-parametrised systems in a compositional way.

Bounds2 is a tool that enables parametrised verification by establishing upper bounds, i.e., cut-offs, for the values of parameters such that if there is a bug in an implementation instance with a parameter value greater than the cut-off, then there is an analogous bug in an implementation instance where the values of the parameters are within the cut-offs. When using Bounds2, implementations and specifications are composed of labelled transitions systems (LTSs) with explicit input and output events by using parallel composition and hiding. We can also use several kinds of parameters: types represent the sets of the identifiers of replicated components or the sets of data values of an arbitrary size, typed variables refer to the identities of individual components or data values and relation symbols represent binary relations over replicated processes. Correctness is understood either as a refinement, which can be trace inclusion [1] or alternating simulation [2], or the compatibility of the components of the implementation [2]. Hence, Bounds2 enables compositional reasoning, too. 
The tool consists of two parts. The instance generator determines cut-offs for the types, computes the allowed parameter values up to the cut-offs and outputs the corresponding finite state verification tasks. It can also apply a limited form of abstraction if the cut-offs cannot be determined without. After that, the outputted instances are verified by an instance checker specific to the notion of correctness. Trace refinement and compatibility checkers exploit the refinement checker FDR2 [1] to verify the instances, the alternating simulation checker makes use of the MIO Workbench [3] refinement checker, too. Bounds2 is publicly available at [4].

\section{The Description Language}

To get a grasp of the CSP-based (Communicating Sequential Processes [1]) formalism used by Bounds2, consider an arbitrary number of processes competing for an access to an arbitrary number of shared variables that store values of a parametric type. Each variable should be written to in a mutually exclusive way, so the interface of the shared variables is formalised as follows:

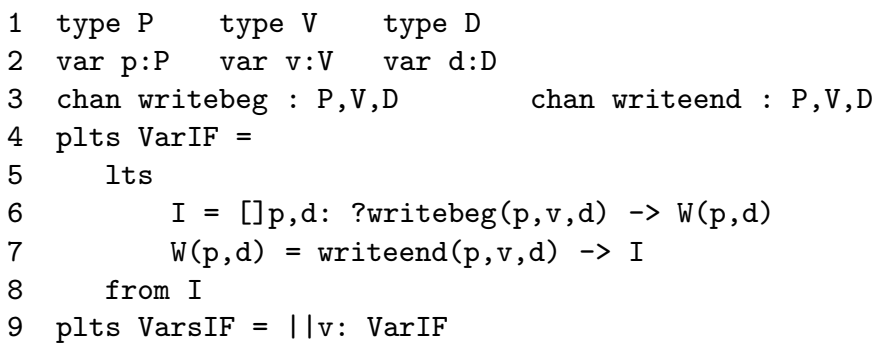

Types $\mathrm{P}$ and $\mathrm{V}$ represent the set of the identifiers of processes and variables, respectively, and $\mathrm{D}$ denotes the domain of the shared variables. Variables $\mathrm{p}, \mathrm{V}$ and $d$ are used to refer to an individual process, a shared variable and a value of a shared variable, respectively. The event $\operatorname{writebeg}(p, v, d)$ (writeend $(p, v, d)$ ) denotes that the process $\mathrm{p}$ starts (is finished with) writing the value $\mathrm{d}$ to the shared variable v. The input events are marked by ?, the other events are outputs. A parametrised LTS (PLTS) VarIF captures the interface of a shared variable $\mathrm{v}$ : only one process can access $\mathrm{v}$ at a time. As we let $\mathrm{v}$ to range over all identifiers of shared variables and compose the instances of VarIF in parallel, we obtain the PLTS VarsIF which captures the joint interface of all the shared variables.

Suppose that we also have an implementation VarImpl of the variable interface, the interface PrIF of a process $\mathrm{p}$ and the alphabet PrAlph of PrIF without the write events. In order to check that (a) all the variable and process interfaces are compatible, i.e., they can co-operate in some environment such that whenever an output is sent, it is matched by an input, (b) the implementation of the variable refines its interface and (c) no two process access the variable simultaneously, we specify the following parametrised verification tasks: 
11 alternating simulation: verify VarImpl against VarIF

12 trace refinement: verify $(|| p: \operatorname{PrIF}) \backslash\left(\left(_{-}\right) \mathrm{p}:\right.$ PrAlph) against VarsIF

We can also define binary relations over parametric types. For example, in order to specify a total order TO in which the processes access the shared variables we could write as follows:

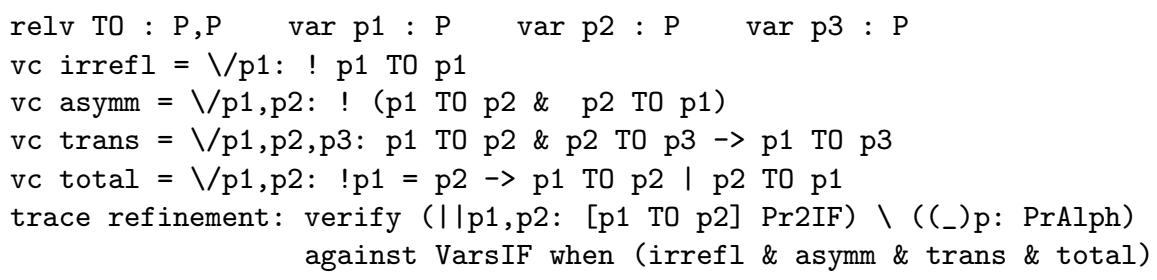

In this case, we need also a PLTS Pr2IF which describes the behaviour of the process interface from the viewpoint of two processes $\mathrm{p} 1$ and $\mathrm{p} 2$ such that $\mathrm{p} 1$ comes before p2 in the total order.

Once we have proved that a system implementation refines its interface specification, we can use the specification, which is usually much smaller, in place of the system implementation in further verification efforts. This is possible since the input formalism of Bounds2 is compositional.

\section{Novel Features}

The first version of Bounds was introduced in [5] and it featured the support for process types, relation symbols and trace refinement (tref). The novelties of Bounds2 introduced here are fourfold.

Sound and/or complete verification. The input language of Bounds2 is Turing complete in its full generality, but there are explicit conditions under which cut-offs can be computed. The main restriction is that each type must classify either as a data type or a process type, but not both. In the above example, $\mathrm{V}$ is thought as a process type since $\mathrm{v}$ is only used in the replicated parallel compositions (line 9) and D is considered a data type since d only occurs in the replicated choices (line 6 ). The variable $\mathrm{p}$ is used both in a replicated choice and a replicated parallel composition (lines 6,10,12), but sometimes, like in this case, Bounds2 can convert replicated choices into replicated parallel compositions, which means that $\mathrm{P}$ is classified as a process type. Depending on the notion of correctness, there are also limitations on non-determinism, hiding and the use of relation symbols. The decidable fragment covers, e.g., the mutual exclusion properties of concurrent systems with shared resources [6 - 8], and if any of the assumptions is removed, parametrised verification becomes undecidable [6 8 ].

All the conditions are checked automatically by the tool. If any of them is violated, the user is asked to provide a cut-off manually. In this case, verification is not sound but we can still detect bugs. Sometimes, the tool can perform an 
over-approximating conversion of replicated choices to replicated parallel compositions. In this case, the verification is sound but false negatives are possible. The exact and over-approximating conversions as well as the possibility to enter a cut-off manually are novel features of Bounds2.

More expressive input language. The main novelty of Bounds2 is a more expressive input language with a larger decidable fragment. This is enabled by the introduction of a replicated choice (data types), the classification of events into inputs and outputs, and two new notions of correctness: compatibility (comp) and alternating simulation (altsim) 2]. A replicated choice adds to expressiveness, since it allows us to express components with a parametrised state space. Earlier, it was only possible to parametrise the number of concurrent components. Distinguishing between input and output events allows us to consider the compatibility of PLTSs representing software interfaces and gives rise to another refinement, the alternating simulation, which is a natural notion of the correctness of software interfaces. However, the support for the alternating simulation is currently not as good as for two other notions of correctness, since the back end alternating simulation checker, MIO Workbench, can only handle relatively small models. The theoretical background of the extensions is described in [7, 8].

Faster operation. Bounds2 determines structural cut-offs for types based on the results in [7, 8]. After that, the tool computes the allowed values for all parameters up to the cut-offs by using a search-tree-based enumeration algorithm described in [5]. Since the values of relation symbols are defined in the universal fragment of first order logic, this is basically equivalent to computing the model class of a first order formula up to the cut-offs.

For the recent version of the tool, we have implemented a version of the search algorithm which is parallelised according to the exploratory partitioning scheme [9]. Initially, computation proceeds sequentially in a breadth-first search manner but when the search tree becomes wide enough (several times wider than the number of processor cores available), the subtrees are processed in parallel. This way, we can ensure that the work load is distributed evenly between threads, since some subtrees are much bigger than the others. Once the values of the parameters up to the cut-offs are computed, Bounds2 generates and outputs the corresponding instances of the system specification and implementation. Also this phase is parallelised in Bounds2 by using the input partitioning scheme [9].

Additionally, the internal data structures are optimised. Previously, all states and events were stored as a string which was highly inefficient. In Bounds2, the strings are first converted into integers for faster analysis and finally back into strings when the instances are outputted.

Improved reduction. The cut-offs computed earlier are rough structural ones. They are fast and easy to compute but they are often far from optimal, especially in the case of data types. Therefore, Bounds2 tries to improve the cut-offs further by analysing the instances up to the structural cut-offs. Basically, the tool discards instances that can be obtained as a composition of smaller ones as 
described in [7, 8]. Also this additional reduction is sound and complete and it is an important enhancement over Bounds1, because the discarded instances are always the biggest ones which are the most expensive to verify.

\section{Experimental Results}

We have made several case studies with Bounds2 and compared its performance against Bounds1 [5]. We have not compared Bounds2 with other parametrised verification tools, since most of them are targeted to low level software with finite data [10-14] whereas our focus is on higher level applications which are not only multithreaded but also object-oriented and component-based. The comparison with other tools would be difficult anyway, since the tools solve a different decidable fragment and we are not aware of any other tools for parametrised refinement checking.

For each system, the table below lists the number of types (typ), relation symbols (rel) and variables (var) used in the model, the notion of correctness (corr) and the structural cut-offs for types. For both versions of Bounds, the number of instances outputted and the running times of the instance generator $\left(t_{\mathrm{G}}\right)$ and the instance checker $\left(t_{\mathrm{C}}\right)$ are reported. For Bounds2, the former running times are given with a single core $\left(t_{\mathrm{G} 1}\right)$ and six cores $\left(t_{\mathrm{G} 6}\right)$ being used. We can see that the cut-offs provided by the tool are often very small and compared with Bounds1, the new version not only has a broader application domain but also operates faster and produces less instances that need to be checked. We can also see that the bottleneck in the verification chain is typically not Bounds2 but the back end finite state verification tool. The experiments were made on a six-core AMD Phenom II with 8GB of memory running Ubuntu 12.04 LTS.

\begin{tabular}{|c|c|c|c|c|c|c|c|c|c|c|c|c|}
\hline \multirow[b]{2}{*}{ system } & \multicolumn{5}{|c|}{ parameters } & \multicolumn{3}{|c|}{ Bounds1 } & \multicolumn{4}{|c|}{ Bounds2 } \\
\hline & typ & rel & var & corr & cut-offs & out & $t_{\mathrm{G}}$ & $t_{\mathrm{C}}$ & out & $t_{\mathrm{G} 1}$ & $t_{\mathrm{G} 6}$ & $t_{\mathrm{C}}$ \\
\hline $\mathrm{HCP}$ & 2 & 0 & 0 & tref & 2,9 & no & suppc & orted & 4 & $0.15 \mathrm{~s}$ & $<0.1 \mathrm{~s}$ & $0.3 \mathrm{~s}$ \\
\hline cache_consistency & 2 & 0 & 0 & tref & 1,13 & no & suppc & rted & 5 & $15 \mathrm{~s}$ & $6 \mathrm{~s}$ & $\overline{9 s}$ \\
\hline tokenring & 1 & 1 & 2 & tref & 4 & 3 & $<0.1 \mathrm{~s}$ & $0.2 \mathrm{~s}$ & 3 & $<0.1 \mathrm{~s}$ & $<0.1 \mathrm{~s}$ & $0.2 \mathrm{~s}$ \\
\hline twotoken & 1 & 2 & 2 & tref & 5 & 30 & $32 \mathrm{~s}$ & $5 \mathrm{~s}$ & 30 & $32 \mathrm{~s}$ & $8 \mathrm{~s}$ & $5 \mathrm{~s}$ \\
\hline miniSRS_comp & 2 & 0 & 0 & comp & 2,1 & no & supp & rrted & 2 & $<0.1 \mathrm{~s}$ & $<0.1 \mathrm{~s}$ & $0.15 \mathrm{~s}$ \\
\hline SRS & 2 & 1 & 0 & tref & 2,3 & 14 & $0.4 \mathrm{~s}$ & $1 \mathrm{~m} 35 \mathrm{~s}$ & 6 & $0.1 \mathrm{~s}$ & $<0.1 \mathrm{~s}$ & $1 \mathrm{~m} 4 \mathrm{~s}$ \\
\hline SRSwithData & 3 & 1 & 0 & tref & $2,3,1$ & no & suppc & rted & 5 & $0.1 \mathrm{~s}$ & $0.1 \mathrm{~s}$ & $1 \mathrm{~m} 4 \mathrm{~s}$ \\
\hline res_io & 2 & 0 & 1 & altsim & 2,1 & $\overline{\text { no }}$ & supp & rted & 2 & $<0.1 \mathrm{~s}$ & $<0.1 \mathrm{~s}$ & $5.7 \mathrm{~s}$ \\
\hline peterson_io & 2 & 0 & 1 & altsim & 2,1 & no & suppc & orted & 1 & $<0.1 \mathrm{~s}$ & $<0.1 \mathrm{~s}$ & $11 \mathrm{~s}$ \\
\hline taDOM2+ & 2 & 2 & 0 & tref & 2,3 & 28 & 11s & $>12 \mathrm{~h}$ & 14 & $6.5 \mathrm{~s}$ & $1.7 \mathrm{~s}$ & $6 \mathrm{~h} 36 \mathrm{~m}$ \\
\hline
\end{tabular}

\section{Conclusions}

Bounds2 is a cut-off-based tool for parametrised verification. The cut-offs provided by the tool are often as good as we can intuitively hope for, which is necessary for practical parametrised verification. The distinctive feature of the 
tool is sound and complete verification with the support for compositional reasoning and the possibility to parametrise both the number of processes and the size of data types as well as the structure of the system. We believe that the tool will be useful in the analysis of multithreaded, component-based, objectoriented software systems, which involve both process and data parameters and where some components may only be available in the interface specification form. Hence, Bounds2 nicely complements other parametrised verification tools most of which are targeted for low level software acting on finite data.

Acknowledgements. We would like to thankfully acknowledge the funding from the SARANA project in the SAFIR 2014 program and the Academy of Finland project 139402.

\section{References}

1. Roscoe, A.W.: Understanding Concurrent Systems. Springer (2010)

2. De Alfaro, L., Henzinger, T.: Interface automata. ACM SIGSOFT Software Engineering Notes 26(5), 109-120 (2001)

3. Bauer, S.S., Mayer, P., Schroeder, A., Hennicker, R.: On weak modal compatibility, refinement, and the MIO workbench. In: Esparza, J., Majumdar, R. (eds.) TACAS 2010. LNCS, vol. 6015, pp. 175-189. Springer, Heidelberg (2010)

4. Siirtola, A.: Bounds website, http://www.cs.hut.fi/u/siirtoa1/bounds

5. Siirtola, A.: Bounds: from parameterised to finite-state verification. In: Caillaud, B., Carmona, J., Hiraishi, K. (eds.) ACSD 2011, pp. 31-35. IEEE (2011)

6. Siirtola, A.: Algorithmic Multiparameterised Verification of Safety Properties. Process Algebraic Approach. PhD thesis, University of Oulu (2010)

7. Siirtola, A., Heljanko, K.: Parametrised compositional verification with multiple process and data types. In: Carmona, J., Lazarescu, M.T., Pietkiewicz-Koutny, M. (eds.) ACSD 2013, pp. 67-76. IEEE (2013)

8. Siirtola, A.: Parametrised interface automata (unpublished draft) (2013), http://www.cs.hut.fi/u/siirtoa1/papers/pia_paper.pdf

9. Grama, A., Gupta, A., Karypis, G., Kumar, V.: Introduction to Parallel Computing. Addison Wesley (2003)

10. Delzanno, G., Raskin, J.-F., Van Begin, L.: Towards the automated verification of multithreaded Java programs. In: Katoen, J.-P., Stevens, P. (eds.) TACAS 2002. LNCS, vol. 2280, pp. 173-187. Springer, Heidelberg (2002)

11. Ghilardi, S., Ranise, S.: Backward reachability of array-based systems by SMT solving: termination and invariant synthesis. Log. Meth. Comput. Sci. 6(4) (2010)

12. Kaiser, A., Kroening, D., Wahl, T.: Dynamic cutoff detection in parameterized concurrent programs. In: Touili, T., Cook, B., Jackson, P. (eds.) CAV 2010. LNCS, vol. 6174, pp. 645-659. Springer, Heidelberg (2010)

13. La Torre, S., Madhusudan, P., Parlato, G.: Model-checking parameterized concurrent programs using linear interfaces. In: Touili, T., Cook, B., Jackson, P. (eds.) CAV 2010. LNCS, vol. 6174, pp. 629-644. Springer, Heidelberg (2010)

14. Yang, Q., Li, M.: A cut-off approach for bounded verification of parameterized systems. In: Kramer, J., Bishop, J., Devanbu, P.T., Uchitel, S. (eds.) ICSE 2010, pp. 345-354. ACM (2010) 